\title{
Vacuum Pressure, Dark Energy, and Dark Matter
}

\author{
Bogusław Broda and Michał Szanecki \\ Department of Theoretical Physics, University of Łódź, Pomorska 149/153, 90-236 Łódź, Poland \\ Correspondence should be addressed to Bogusław Broda, bobroda@uni.lodz.pl
}

Received 3 November 2011; Accepted 27 December 2011

Academic Editors: R. N. Henriksen and M. Sazhin

Copyright ( $) 2011$ B. Broda and M. Szanecki. This is an open access article distributed under the Creative Commons Attribution License, which permits unrestricted use, distribution, and reproduction in any medium, provided the original work is properly cited.

It has been argued that the correct, that is, positive, sign of quantum vacuum energy density, or, more properly, negative sign of quantum vacuum pressure, requires not a very large, and to some extent model-independent, number, for example, $\sim 100$, of additional, undiscovered fundamental bosonic particle species, absent in the standard model. Interpretation of the new particle species in terms of dark matter ones permits to qualitatively, and even quantitatively, connect all the three concepts given in the title.

Dark energy [1-3] and dark matter $[4,5]$ are two main constituents of our universe. Their contribution amounts to almost $96 \%$ of the total energy-mass of the universe. The nature of the both seems to be mysterious but completely different. Another mysterious and elusive constituent of our universe, appearing in theoretical context rather than in a cosmological one, is quantum vacuum [6]. But, recently, mainly due to advances in the Casimir effect, the quantum vacuum is beginning to enter reality [7]. The old idea to identify dark energy and quantum vacuum energy is theoretically very attractive but the main difficulty is to reconcile the values of both energies [8-13]. That is a puzzle. But there is another puzzle, usually not being mentioned. Since, as it is well known, the sign of quantum vacuum energy follows from the statistics of fluctuating fields, the right (positive) sign of vacuum energy corresponds to bosonic modes. But the number of different fermionic particle species prevails in the standard model, and that is the puzzle. Thus, actually, we have the two independent puzzles related to the connection between quantum vacuum energy and dark energy: the puzzle of the huge (absolute) value of quantum vacuum energy density and the puzzle of its sign.

It appears, and this is the main subject of our letter, that it is possible to solve the second puzzle, establishing a link between the issue of dark energy and dark matter. Namely, not a very large, and to some extent model-independent, number of undiscovered bosonic fields should be included in the fundamental set of particle species to obtain the right sign of vacuum energy density. These new species are natural candidates for dark matter particle species.

A typical approach to quantum vacuum energy yields the standard formula $[8]$

$$
\varrho_{\mathrm{vac}}=\frac{1}{2} \int_{0}^{\Lambda_{\mathrm{uv}}} \frac{4 \pi}{(2 \pi \hbar)^{3} c} \sqrt{(m c)^{2}+k^{2}} k^{2} d k,
$$

where $m$ is the mass of a bosonic mode and $k$ is its momentum. For a large ultraviolet (UV) cutoff $\Lambda_{\mathrm{uv}}$ we have approximately

$$
\varrho_{\mathrm{vac}} \approx \frac{1}{(4 \pi)^{2}} \frac{\Lambda_{\mathrm{uv}}{ }^{4}}{\hbar^{2} c} .
$$

Setting $\Lambda_{\mathrm{uv}}=\Lambda_{\mathrm{P}}$, where the Planck momentum

$$
\Lambda_{\mathrm{P}}=\sqrt{\frac{\hbar c^{3}}{G}} \approx 6.5 \mathrm{~kg} \mathrm{~m} / \mathrm{s}
$$

and $G$ is the Newton gravitational constant, we obtain the "(in)famous" value (formula)

$$
\varrho_{\mathrm{vac}} \approx \frac{c^{5}}{(4 \pi)^{2} \hbar G^{2}} \approx 3.4 \times 10^{94} \mathrm{~kg} / \mathrm{m}^{3} \approx \frac{M_{\mathrm{P}}}{L_{\mathrm{P}}^{3}},
$$

where $M_{\mathrm{P}}$ and $L_{\mathrm{P}}$ are the Planck mass and length, respectively. The fame of this formula rivals its absurdity. Not only is (4) some $10^{120}$ times greater than expected but evidently 
the sign is not as expected. For fermionic modes the sign of (4) will be reversed! Therefore, for presently known contents of fundamental set of fields (with prevalent fermionic modes) the sign will be wrong. Consequently, new bosonic species are urgently being looked for.

One should emphasize that the difficulty with the sign seems to be independent of the approach to the issue of the huge value of quantum vacuum energy. In other words, additional bosonic modes are presumably unavoidable provided quantum vacuum is supposed to have something to do with dark energy or, at least, with reality. Taking for granted that new bosonic particle species should enter the set of fundamental fields any further estimate of their number could already depend on the assumed model of dark energy in the framework of the idea of quantum vacuum.

In $[14,15]$, we have proposed a phenomenologically promising approach to solve the puzzle of the huge (absolute) value of "the quantum vacuum energy density." In the framework of our approach the (absolute) value of the "vacuum energy density" of a single mode is of the order

$$
\varrho \sim 0.01 \varrho_{\exp }
$$

where $\varrho_{\exp }$ is the experimental value of the energy density of dark energy. Thus, (5) gives the result for a single mode. Obviously, the relation (5) is model dependent. In our approach $[14,15]$ it is just $(5)$.

Strictly speaking, the formula yielding the result (5), that is, the lagrangian density $[14,15]$

$$
\mp \frac{1}{4} \frac{1}{(4 \pi)^{2} G} \frac{1}{2}(1-q) H^{2}
$$

where the upper and lower signs correspond to a bosonic and fermionic mode, respectively, $q$ is the present deceleration parameter, and $H$ is the present Hubble expansion rate, corresponds to the pressure rather than to the energy density.

In fact, the diagonal part of the energy-momentum tensor,

$$
T_{\mu \nu}=\frac{\partial \mathcal{L}}{\partial \partial^{\mu} \phi} \partial_{\nu} \phi-g_{\mu \nu} \mathcal{L}
$$

reduces to

$$
T_{00} \equiv \varrho=-g_{00} \mathcal{L}, \quad \text { for } \partial_{0} \phi=0,
$$

or

$$
T_{i i} \equiv p=-g_{i i} \mathcal{L}, \quad \text { for } \partial_{i} \phi=0,
$$

where $c=1$ and the signature of the metric is $(+,-,-,-)$. Our case corresponds obviously to the second possibility, that is, (9), because our fields are homogeneous $\left(\partial_{i} \phi=0\right)$ but time dependent (Friedmann-Lemaitre-Robertson-Walker cosmological model).

Coming back to our model

$$
\mp \frac{1}{4} \frac{1}{(4 \pi)^{2} G} \frac{1}{2}(1-q) H^{2} \approx \mathcal{L}=-\frac{1}{g_{i i}} p,
$$

and therefore

$$
p \approx \mp \frac{1}{4} \frac{1}{(4 \pi)^{2} G} \frac{1}{2}(1-q) H^{2}
$$

which still conforms with our description of dark energy in terms of quantum vacuum. For example, assuming temporarily an ad hoc barotropic relation

$$
p=w \varrho, \quad w=-1
$$

we simply obtain

$$
\varrho \approx \pm \frac{1}{128 \pi^{2} G}(1-q) H^{2}
$$

But (11) (for pressure) is more fundamental than (13) (for energy density) because no equation of state needs to be presumed.

Consequently, as a next step, we should collect contributions, of the type estimated by us, coming from all fundamental physical modes. We can proceed in the spirit of the philosophy of quantum- (vacuum-) induced interactions (see, e.g., [16-18]). Thus, the total pressure, coming from all fundamental modes, is according to (11) of the order

$$
p \approx-\frac{N_{0}}{128 \pi^{2} G}(1-q) H^{2}
$$

and $\varrho_{\exp } \approx N_{0} \varrho$, where $N_{0}$ is the "alternated sum" of the fundamental modes. Namely, we define

$$
N_{0} \equiv n_{B}-n_{F}=\sum_{k=0}(-)^{k} n_{k / 2}
$$

where $n_{B}$ and $n_{F}$ are the number of fundamental bosonic and fermionic modes, respectively. By virtue of the spin-statistics theorem we can rewrite $N_{0}$ in terms of spin degrees of freedom, that is, $n_{0}, n_{1 / 2}, n_{1}$, and so forth. One should note that the particular case $n_{B}=n_{F}$ corresponds to supersymmetry.

Recapitulating, the puzzle related to quantum vacuum fluctuations consists in getting under control of the huge value of the vacuum energy density, strictly speaking, from our point of view, the (absolute) value of the pressure. Once the value becomes reasonable in its size, the sign puzzle emerges. The sign assumes the expected value, for example, "minus" in (14), only provided the number of bosonic fundamental modes is prevalent.

We would like to stress, once more, that the conclusion concerning bosonic species is not specific to our model of dark energy, because only bosonic modes give contributions huge or moderate but with right signs. Since the standard model contains greater number of fermionic particle species (leptons and quarks) than bosonic ones (mainly, gauge fields), we can conclude that there is a missing number of invisible bosonic modes. The bosonic modes do not enter the standard model but they must interact gravitationally. Therefore, they are appropriate and natural candidates to the role of dark matter particles. 
More concretely, for the standard model

$$
\begin{aligned}
N_{0} & =[4]-2 \cdot 3 \cdot(3+3 \cdot 4)+2 \cdot(1+3+8+[1]) \\
& =-(63 \pm 3),
\end{aligned}
$$

where in the first bracket we have included, as yet not discovered, Higgs modes, next there are leptons and quarks (2 spins $\times 3$ families $\times(3$ leptons of the both helicities +3 colors $\times 4$ quarks of the both helicities)), and, finally, gauge fields ( 2 spins $\times$ (photon +3 weak bosons +8 gluons $)$ ) with graviton in the last bracket. From (14) and (16) (see, also (5) for a numeric value) it follows that the lacking number of bosonic modes is of the order $n_{B} \sim 100$. This number is model dependent but the conclusion is not.

Any possible, alternative, and independent solution of the puzzle of the huge (absolute) value of quantum vacuum energy density also would require additional bosonic species but their number could vary. For example, any potential solution directly yielding the right (absolute) value of the quantum vacuum energy density, meaning that the correct (absolute) value is recovered for a single mode, would require a tuning between the number of bosonic and fermionic species, that is, $n_{B}-n_{F} \sim 1$.

Certainly, it would be very advantageous to present an alternative and independent estimate of the number of the lacking fundamental bosonic modes $n_{B}$. To this end we will make use of the proposal given in [18], concerning quantum vacuum-induced gravitational action and quantum vacuuminduced gravitational (black-hole) thermodynamical entropy. Assuming the number and kind of fundamental modes given in (16), we get $n_{B} \sim 40$ to properly induce the gravitational action (see equation (8) in [18]), and $n_{B} \sim 200$ to properly induce the gravitational (black-hole) thermodynamical entropy (see equation (2) in [18]). Taking into account an approximate character of our reasoning one should admit that it qualitatively agrees with our present dark energy estimate, that is, $n_{B} \sim 100$.

In this paper, we have proposed a consistent connection between the following three concepts: quantum vacuum pressure, dark energy, and dark matter. First of all, we have shown that, in the context of lagrangian approach, the quantum pressure naturally replaces the notion of quantum vacuum energy density. Next, we have argued that, independently of an actual model of dark energy, additional, undiscovered fundamental bosonic particle species are necessary. Finally, applying our earlier model of dark energy [14, 15] we have estimated the number of the fundamental bosonic particle species, namely, $n_{B} \sim 100$. Amazingly, it agrees with the estimates being yielded by induced gravity [18].

\section{Acknowledgments}

This work has been supported by the University of Łódź grant and LFPPI network.

\section{References}

[1] A. G. Riess, A. V. Filippenko, P. Challis et al., "Observational evidence from supernovae for an accelerating universe and a cosmological constant," The Astronomical Journal, vol. 116, no. 3, pp. 1009-1038, 1998.

[2] S. Perlmutter, G. Aldering, G. Goldhaber et al., "Measurements of $\Omega$ and $\Lambda$ from 42 high-redshift supernovae," The Astrophysical Journal, vol. 517, pp. 565-586, 1999.

[3] E. J. Copeland, M. Sami, and S. Tsujikawa, "Dynamics of dark energy," International Journal of Modern Physics D, vol. 15, no. 11, pp. 1753-1936, 2006.

[4] G. Bertone, D. Hooper, and J. Silk, "Particle dark matter: evidence, candidates and constraints," Physics Reports, vol. 405, no. 5-6, pp. 279-390, 2005.

[5] N. Fornengo, "Status and perspectives of indirect and direct dark matter searches," Advances in Space Research, vol. 41, pp. 2010-2018, 2007.

[6] G. E. Volovik, "Vacuum energy: myths and reality," International Journal of Modern Physics D, vol. 15, no. 12, pp. 19872010, 2006.

[7] R. L. Jaffe, "Casimir effect and the quantum vacuum," Physical Review D, vol. 72, no. 2, Article ID 021301, 2005.

[8] S. Weinberg, "The cosmological constant problem," Reviews of Modern Physics, vol. 61, no. 1, pp. 1-23, 1989.

[9] S. M. Carroll, "The cosmological constant," Living Reviews in Relativity, vol. 4, pp. 1-78, 2001.

[10] T. Padmanabhan, "Cosmological constant - the weight of the vacuum," Physics Reports, vol. 380, no. 5-6, pp. 235-320, 2003.

[11] T. Padmanabhan, "Dark energy: mystery of the millennium," in Proceedings of the Albert Einstein Century International Conference, vol. 861 of AIP Conference Proceedings, pp. 179196, Paris, France, 2005.

[12] Y. B. Zel'dovich, "Cosmological constant and elementary particles," Letters to Jounal of Experimental and Theoretical Physics, vol. 6, no. 9, pp. 316-317, 1967, Translated from Zhurnal Eksperimentalnoi $i$ Teoreticheskoi Fiziki, vol. 6, pp. 883-884, 1967.

[13] S. Nobbenhuis, "Categorizing different approaches to the cosmological constant problem," Foundations of Physics, vol. 36, no. 5, pp. 613-680, 2006.

[14] B. Broda, P. Bronowski, M. Ostrowski, and M. Szanecki, "Vacuum driven accelerated expansion," Annalen der Physik, vol. 17, no. 11, pp. 855-863, 2008.

[15] B. Broda and M. Szanecki, "Quantum vacuum and accelerated expansion," in Dark Energy and Dark Matter: Observations, Experiments and Theories, E. Pécontal, T. Buchert, Ph. di Stefano, and Y. Copin, Eds., vol. 36 of EAS Publications Series, pp. 167-171, 2009.

[16] A. D. Sakharov, "Vacuum quantum fluctuations in curved space and the theory of gravitation," General Relativity and Gravitation, vol. 32, pp. 365-367, 2000, Translated from Doklady Akademii Nauk SSSR, vol. 170, pp. 70-71, 1967.

[17] Y. B. Zeldovich, "Interpretation of electrodynamics as a consequence of quantum theory," ZhETF Pis ma Redaktsiiu, vol. 6, pp. 922-925, 1967, Letters to Jounal of Experimental and Theoretical Physics, vol. 6, pp. 345-347, 1967.

[18] B. Broda and M. Szanecki, "Induced gravity and gauge interactions revisited," Physics Letters B, vol. 674, no. 1, pp. 64-68, 2009. 

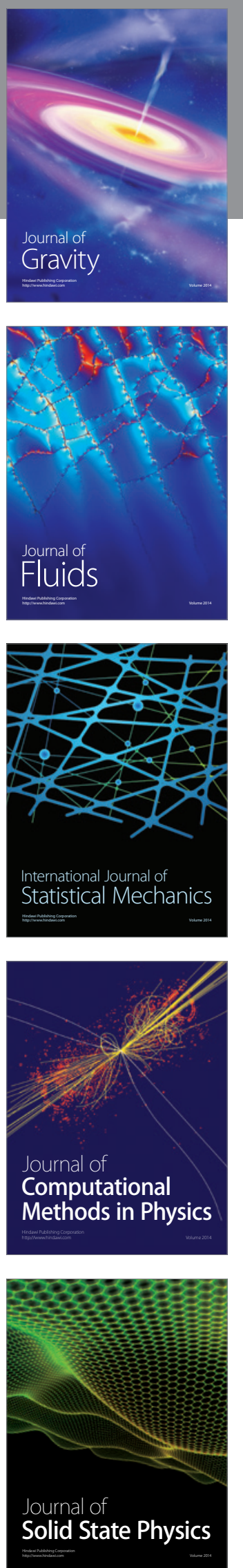

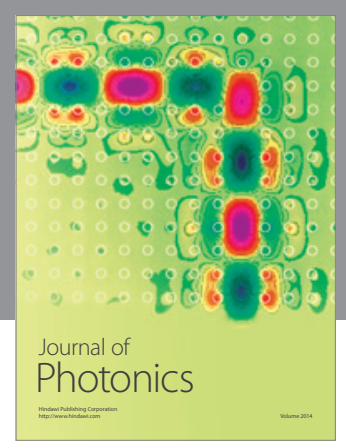

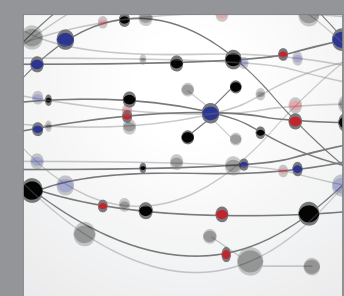

The Scientific World Journal
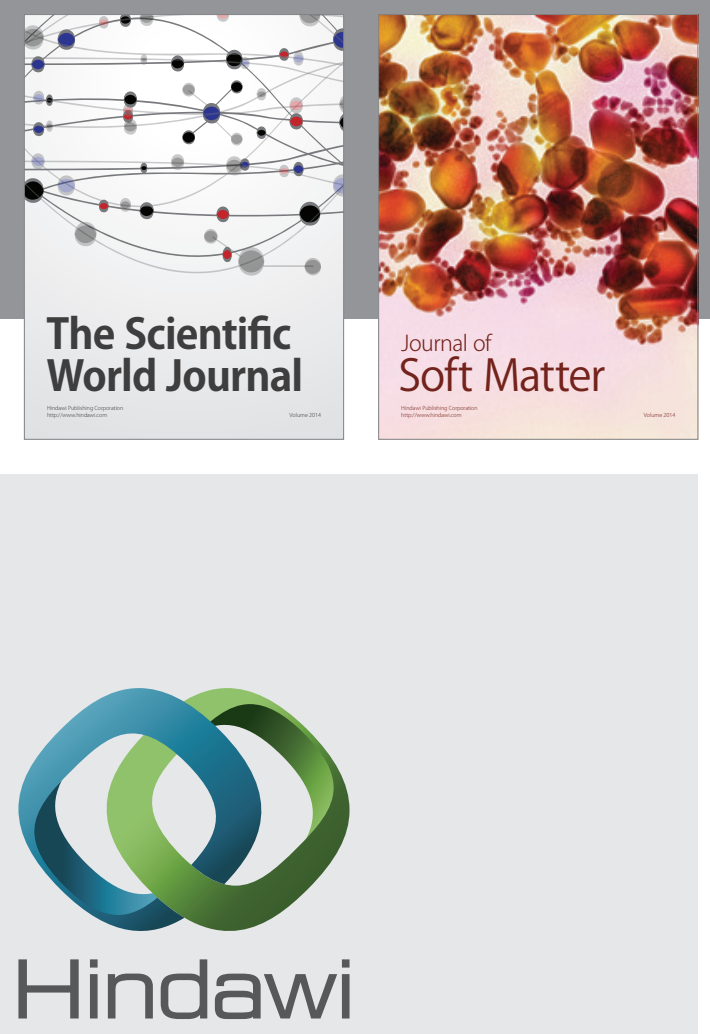

Submit your manuscripts at

http://www.hindawi.com
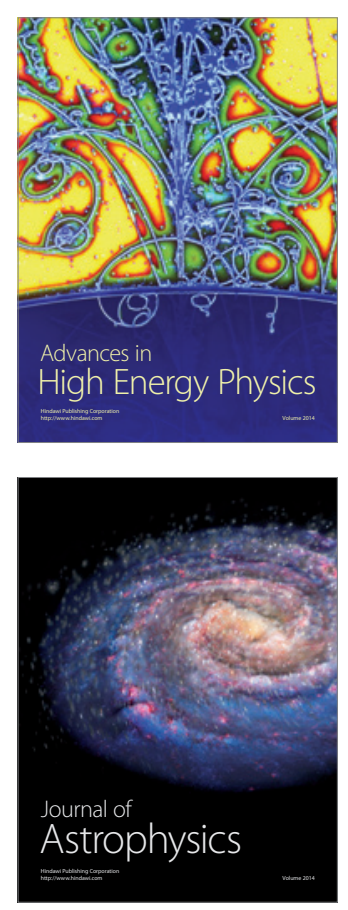
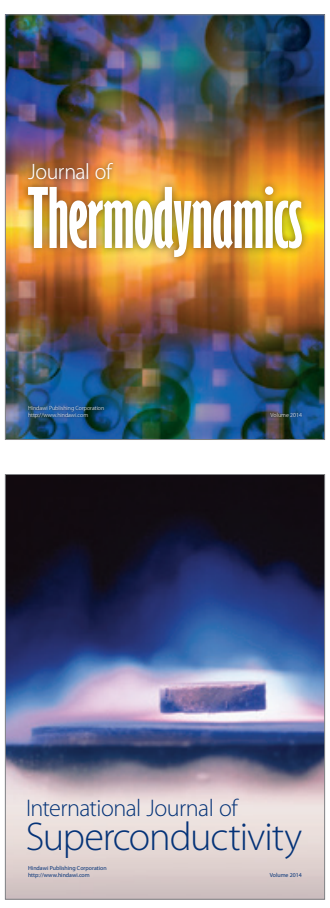
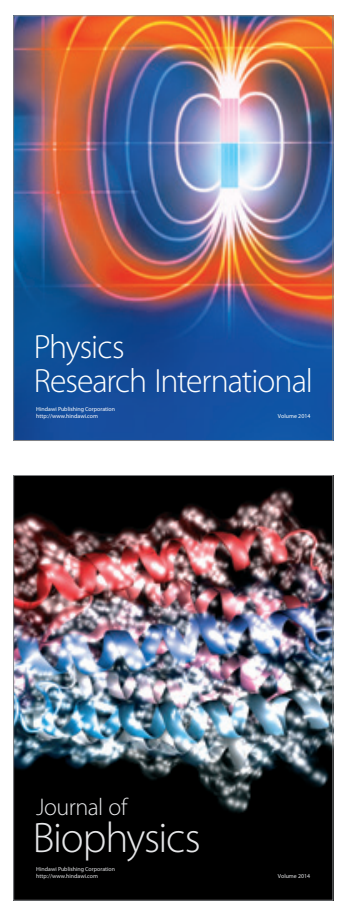
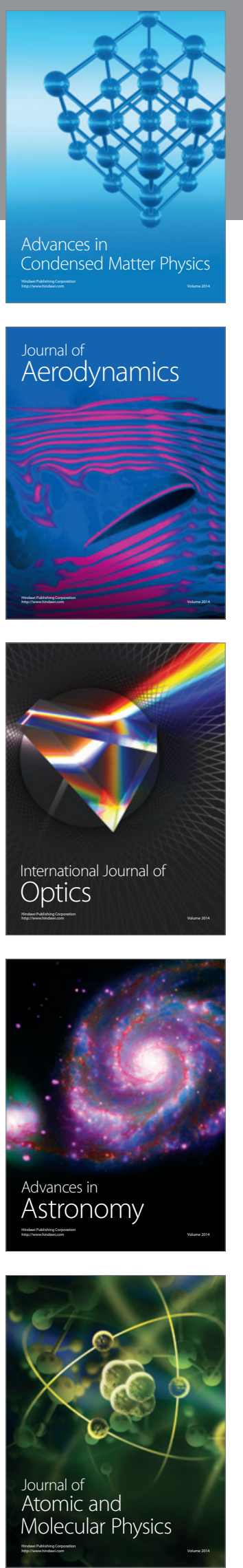